\title{
Synergy Between the University of Louisana at Monroe and the Louisiana Drug Utilization Review Board
}

$\mathrm{T}$ he University of Louisiana at Monroe (ULM) College of Pharmacy, the only state-supported pharmacy school in Louisiana, began its relationship with the Louisiana Department of Health and Hospitals (DHH) about 15 years ago. Over time, the contract has expanded, and ULM now works with $\mathrm{DHH}$ in several key areas

ULM began by providing consulting services to the Louisiana Medicaid Drug Utilization Review (LaDUR) Board in 1990. At that time, there was no online adjudication of claims, and quality and cost-control efforts focused on retrospective review. In 1996, the state developed its own pharmacy benefit management system and instituted online adjudication of claims. Prospective drug-utilization review (DUR) criteria were developed and implemented. In both prospective and retrospective DUR, ULM College of Pharmacy faculty members are available to assist in developing criteria, to research therapeutics, to analyze utilization, and to report to the LaDUR Board.

To keep Medicaid providers informed of policy and reimbursement procedural changes, the DHH publishes the Louisiana Medicaid Provider Update. ULM faculty members contribute educational articles to each issue of this bimonthly publication. Usually these articles are written by clinical faculty and discuss current pharmacotherapeutic guidelines. Recent articles include: "Polypharmacy in the Elderly," "Depression in the Elderly," "Management of Cancer Pain," and "Congestive Heart Failure: A Review of Drug Therapy." Occasionally articles will report results of analyses, such as "A Look at the Louisiana Medicaid Lock-in Program" and "Prescription Drug Utilization for the Treatment of Erectile Dysfunction in the Louisiana Medicaid Program."

In 1997, the ULM School of Pharmacy expanded its services to the Louisiana Medicaid program by initiating population-wide disease management (DM) programs. These programs have never focused on cost savings, but have the ultimate goal of improving patient outcomes. To date, the DM efforts have been population-wide educational mailings to practitioners and Medicaid recipients. All recipients who have been diagnosed with the targeted disease are included in the disease-management efforts regardless of severity or utilization. The following disease states were initially selected for DM:

Diabetes. DM efforts focused on glycosylated hemoglobin (HgAlc) testing and increasing the number of recipients with a diagnosis of diabetes who received at least one HgAlc a year. Statewide data analysis showed an increase in continuously eligible recipients who received at least one HgAlc test per year from $18.78 \%$ to $19.97 \%$.

Asthma. Asthma DM emphasized regular use of a peak flow meter, control of environmental triggers, and appropriate drug therapy. Over the past three years, inpatient admissions and emergency department visits declined while prescription drug use increased. Total costs per patient, however, declined.

Arthritis. Arthritis DM focused on reducing gastrointestinal adverse events as a result of NSAID therapy.

Gastrointestinal. Gastrointestinal DM efforts seek to increase the number of ulcer patients who have received an $\mathrm{H}$. pylori test.

The results of the latter two programs are being evaluated.

DM efforts have been initiated in other disease states as well, including depression, $\mathrm{AD} / \mathrm{HD}$, hyperlipidemia, and congestive heart failure. These DM efforts are in the early stages of development and/or implementation. The claims data provided to ULM to monitor recipient utilization in these disease areas are also available to a limited extent to students for independent projects such as dissertation research, provided approval for such research has been obtained from DHH

ULM has also assisted the DHH in other ways. For the Medicaid program, we are studying concurrent use of proton pump inhibitors and histamine-2 antagonists and following this use longitudinally to determine whether our educational efforts have reduced this duplicative therapy. Another area of concern to $\mathrm{DHH}$ is adverse drug events resulting from drug interactions involving scheduled drugs. ULM faculty are investigating this subject from a pharmacological perspective.

ULM faculty also served on the Louisiana Diabetes Task Force evaluating the impact of diabetes education on the quality of life of diabetic patients and determining barriers to better diabetes care as perceived by patients and health care practitioners.

ULM has also used claims data to study the effect of DHH policy, such as limiting coverage of drugs for erectile dysfunction to six units per month, on Medicaid recipient utilization.

Over the past few years, the cooperation and relationship between the Louisiana Department of Health and Hospitals and the University of Louisiana at Monroe has developed and expanded. We at ULM College of Pharmacy appreciate this relationship and believe that, with the increasing importance of pharmaceutical agents in disease treatment and the rapidity with which new agents are introduced into the market, the role of pharmacy in patient care has just begun its upward trajectory. We would like to see these collaborative efforts between the ULM College of Pharmacy and DHH have a significant positive impact on the health of the Louisiana Medicaid population and on the health of all citizens of the state.

Sandra Blake, Ph.D., Assistant Professor of Pharmacy Administration, University of Louisiana at Monroe College of Pharmacy, Monroe, Louisiana. 